\title{
Persistent Cannabis Abuse and Risk for Hospitalization for Acute Pancreatitis: A Cross- Sectional Study in United States Hospitals
}

Renu Bhandari ${ }^{1}$, Siddharth Gupta ${ }^{2}$, Karnav Modi ${ }^{3}$, Maharshi R. Raval ${ }^{3}$, Hajara Joundi ${ }^{4}$, Jeet R. Patel ${ }^{5}$, Amanpreet K. Pannu ${ }^{6}$, Prerna Sharma ${ }^{7}$

1. Medicine, Manipal College of Medical Sciences, Kaski, NPL 2. Internal Medicine, Sri Guru Ram Das Institute of Medical Sciences and Research, Amritsar, IND 3. Internal Medicine, Byramjee Jeejeebhoy (BJ) Medical College, Ahmedabad, IND 4. Internal Medicine, University Cadi Ayyad, Faculty of Medicine and Pharmacy, Marrakech, MAR 5. Internal Medicine: Pediatrics, Byramjee Jeejeebhoy (BJ) Medical College, Ahmedabad, IND 6. Medicine, Sri Guru Ram Das University of Health Sciences, Amritsar, IND 7. Psychiatry, Government Medical College, Amritsar, IND

Corresponding author: Prerna Sharma, perusharma90@gmail.com

\section{Abstract \\ Objectives}

To explore the independent association between cannabis abuse and subsequent hospitalizations for acute pancreatitis (AP) and delineate the demographic differences among AP in patients with and without persistent cannabis abuse.

\section{Methods}

We conducted a retrospective cross-sectional study using the nationwide inpatient sample and included $50,444,133$ patients (age 18-50 years) with a primary discharge diagnosis for medical illnesses and further grouped by presence of AP $(\mathrm{N}=666,248)$. We used the logistic regression model to measure the odds ratio (OR) of the association between cannabis abuse and hospitalization for AP and adjusted it for demographic confounders and comorbid risk factors.

\section{Results}

Cannabis abuse significantly increases the odds for AP-related hospitalization (OR 2.12, P $<0.001)$. When the regression model was controlled for potential risk factors (gall stones, cystic fibrosis, hypertriglyceridemia, hypercalcemia, hyperparathyroidism, abdominal surgeries, tobacco abuse, and alcohol abuse), cannabis abuse did not increase the odds for AP-related hospitalization (OR 0.72, P <0.001) due to the significant effect caused by gallstones (OR 30.98, $\mathrm{P}<0.001$ ) and alcohol abuse (OR 12.69, $\mathrm{P}<0.001)$. AP inpatients with cannabis abuse were younger compared to non-cannabis abusers (mean age, 35.7 vs. 37.9 years), and majorly male (70.9\% vs. $53.8 \%)$. AP was considerably more prevalent in whites (60.6\%), followed by blacks (18.3\%) and Hispanics (15.2\%).

Review began 05/27/2021 Review ended 06/03/2021 Published 06/11/2021

\section{๑) Copyright 2021}

Bhandari et al. This is an open access article distributed under the terms of the Creative Commons Attribution License CC-BY 4.0., which permits unrestricted use, distribution, and reproduction in any medium, provided the original author and source are credited.

\section{Conclusion}

Cannabis abuse increased the unadjusted odds for AP-related hospitalization by two times, but after controlling for potential risk factors the adjusted odds of association significantly reduced. Cannabisinduced AP can be treated if a problematic recreational cannabis use pattern is discontinued at an earlier stage. Therefore, awareness campaigns and early supportive therapy among cannabis abusers might help diagnose and treat the comorbidity and improve the quality of life.

Categories: Internal Medicine, Gastroenterology, Public Health

Keywords: acute pancreatitis, cannabis use disorder, cannabis research, marijuana abuse, national inpatient sample, risk-factors

\section{Introduction}

Acute pancreatitis (AP) is a severe inflammatory condition associated with significant morbidity and mortality. It is responsible for about 275,000 hospital admissions every year in the United States (US), costing more than 2.5 billion dollars annually [1]. Overall, the incidence is 34 per 100,000 general population per year and affects men and women equally. The incidence of AP is increasing and compared to the European and Western Pacific regions, the incidence and mortality are significantly higher in the US [2-3].

In the US, cannabis is the most commonly used illicit substance, and its use has increased from $8.2 \%$ to $14.5 \%$ of the total US population from 1992 to 2016 [4]. Synthetic cannabinoids are more common in younger users, as they are inexpensive and easily accessible. In addition, they have much higher efficacy and affinity to bind to cannabinoid receptors as compared to the natural forms. Their appeal is further enhanced to the 
younger demographic, as they are also challenging to identify with routine drug screenings [5].

According to the substance abuse and mental health services administration, cannabis is the most commonly abused drug among those who drink, with the exception of tobacco. Among current drinkers, $11.4 \%$ reported cannabis use in the past 12 months; 3.9\% reported concurrent cannabis use (concurrent users) and $7.5 \%$ of drinkers reported they usually or sometimes used cannabis with alcohol (simultaneous users) [6].

The relationship between cannabis and pancreatitis has always been a topic of debate, as there is limited literature. Some studies report cannabis causing pancreatitis while other studies support the protective effect of cannabis in alcohol-induced pancreatitis [7]. Approximately $20 \%$ of AP cases are classified as idiopathic. The diagnosis of cannabis-associated pancreatitis is made after an extensive workup fails to identify any other cause. This accounts for a small fraction of idiopathic cases of AP. Cannabis use is associated with nine percent of idiopathic cases and ten percent of all first episodes of AP [8]. So, we conducted an inpatient study to explore the independent association of cannabis abuse and hospitalizations for AP after controlling for potential risk factors and demographic confounders. Furthermore, we delineated the demographic differences among AP in patients with versus without cannabis abuse.

\section{Materials And Methods}

We conducted a retrospective cross-sectional study using the Nationwide Inpatient Sample (NIS, 2010 to 2014), the largest database of hospital stays in the US. It is a reliable data source for epidemiological estimates that involve the risk factors associated with diseases [9]. We included 50,444,133 patients (age 1850 years) with a primary discharge diagnosis for medical conditions and were further grouped by the presence of AP $(\mathrm{N}=666,248)$.

The demographic variables studied in this analysis are age, sex, and race [10]. The existing risk factors for AP were defined based on current literature and included gall stones, cystic fibrosis, hypertriglyceridemia, hypercalcemia, hyperparathyroidism, abdominal surgeries, tobacco abuse, alcohol abuse, and cannabis abuse.

We compared the non-AP and AP groups using bivariate analysis using descriptive statistics to delineate the differences in demographics and comorbid risk factors. The first model of logistic regression analyses was conducted to determine the odds ratio (OR) of association between cannabis abuse and hospitalization for $\mathrm{AP}$, and in the next model, it was adjusted for demographics and comorbid risk factors (gall stones, cystic fibrosis, hypertriglyceridemia, hypercalcemia, hyperparathyroidism, abdominal surgeries, tobacco abuse, and alcohol abuse). Next, we conducted the bivariate analysis using descriptive statistics and the $P$ values were generated using the Pearson's chi-square test to compare the demographics in cannabis users and nonusers hospitalized for AP. All statistical analyses were performed in the statistical package for the social sciences (SPSS) version 26.0 (IBM Corp., Armonk, NY) with a statistical significance set a priori at $P$ value $<0.01$.

Unique patient identifiers were used to protect the patient identity. So the use of the NIS, according to the US department of health and human services, does not require approval from an institutional review board [9].

\section{Results}

The prevalence of AP in the medical inpatient population was $1.32 \%(666,248$ out of $50,444,133)$ and were older than non-AP inpatients (mean age, 37.8 vs. 33.9 years). Males had higher odds (OR 1.54; 95 CI 1.537 $1.555)$ compared to females for being hospitalized for AP. AP was considerably more prevalent in whites (60.6\%), followed by blacks (18.3\%) and Hispanics (15.2\%).

In our study, there exists a statistically significant association between certain risk factors and AP. Patients with gallstones had the highest odds of AP hospitalization (OR 30.98; 95\% CI 30.699 - 31.253), followed by alcohol abuse (OR 12.69; 95\% CI 12.613 - 12.776), cystic fibrosis (OR 2.55; 95\% CI 2.417 - 2.697), hypertriglyceridemia (OR 2.53; $95 \%$ CI $2.507-2.542$ ), and hypercalcemia (OR 2.26; 95\% CI $2.180-2.340$ ). The previous history of abdominal surgery was not shown to have a statistically significant risk for AP hospitalization.

Cannabis abuse significantly increased the odds for AP hospitalization (OR 2.12; 95\% CI 2.095 - 2.149). But when the regression model was adjusted for demographics and other potential risk factors, cannabis abuse did not increase the odds for AP hospitalization (OR 0.72; 95\% CI 0.713 - 0.734), as shown in Table 1. 


\section{Cureus}

\begin{tabular}{|c|c|c|c|c|c|}
\hline \multirow{2}{*}{ Variables } & \multicolumn{2}{|c|}{ Acute pancreatitis } & \multicolumn{3}{|c|}{ Logistic regression model } \\
\hline & $(-)^{\star}$ in \% & $(+)^{\star}$ in $\%$ & OR & $95 \% \mathrm{Cl}$ & P-value \\
\hline Total Patients & 49777885 & 666,248 & - & - & - \\
\hline Mean age (SD), in years & $33.9(9.3)$ & $37.8(8.6)$ & 1.00 & $1.003-1.004$ & $<0.001$ \\
\hline \multicolumn{6}{|l|}{ Gender } \\
\hline Male & 27.5 & 54.5 & 1.54 & $1.537-1.555$ & $<0.001$ \\
\hline Female & 72.5 & 45.5 & \multicolumn{3}{|c|}{ Reference } \\
\hline \multicolumn{6}{|l|}{ Race } \\
\hline White & 56.1 & 60.6 & \multicolumn{3}{|c|}{ Reference } \\
\hline Black & 19.0 & 18.3 & 0.93 & $0.925-0.938$ & $<0.001$ \\
\hline Hispanic & 16.7 & 15.2 & 0.95 & $0.940-0.955$ & $<0.001$ \\
\hline Other & 8.1 & 5.9 & 0.81 & $0.799-0.817$ & $<0.001$ \\
\hline \multicolumn{6}{|l|}{ Risk factors } \\
\hline No risk factor & - & - & \multicolumn{3}{|c|}{ Reference } \\
\hline Gall stones & 0.8 & 16.0 & 30.98 & $30.699-31.253$ & $<0.001$ \\
\hline Cystic fibrosis & 0.1 & 0.2 & 2.55 & $2.417-2.697$ & $<0.001$ \\
\hline Hypertriglyceridemia & 8.0 & 21.1 & 2.53 & $2.507-2.542$ & $<0.001$ \\
\hline Hypercalcemia & 0.2 & 0.6 & 2.26 & $2.180-2.340$ & $<0.001$ \\
\hline Hyperparathyroidism & 0.1 & 0.1 & 1.31 & $1.221-1.406$ & $<0.001$ \\
\hline Abdominal surgeries & 13.1 & 20.7 & 0.99 & $0.987-1.001$ & 0.086 \\
\hline Tobacco abuse & 14.5 & 37.1 & 1.61 & $1.597-1.617$ & $<0.001$ \\
\hline Alcohol abuse & 3.7 & 38.0 & 12.69 & $12.613-12.776$ & $<0.001$ \\
\hline Cannabis abuse & 1.8 & 3.8 & 0.72 & $0.713-0.734$ & $<0.001$ \\
\hline
\end{tabular}

\section{TABLE 1: Regression analysis for acute pancreatitis hospitalization}

${ }^{*}(-)$ refers to non-acute pancreatitis and $(+)$ refers to acute pancreatitis groups.

OR: odds ratio; Cl: confidence interval; SD: standard deviation.

Approximately 3.8\% ( $\mathrm{N}=25,478$ ) of 666,248 AP inpatients were cannabis abusers. AP inpatients with

cannabis abuse were younger as compared to non-cannabis abusers (mean age, 35.7 vs. 37.9 years). A higher proportion of cannabis abusers were male ( $70.9 \%$ vs. $53.8 \%$ in non-cannabis abusers) and blacks ( $32.6 \%$ vs. $17.7 \%$ in non-cannabis abusers) as shown in Table 2. 


\section{Cureus}

\begin{tabular}{|c|c|c|c|}
\hline \multirow{2}{*}{ Variables } & \multicolumn{2}{|c|}{ Cannabis abuse } & \multirow{2}{*}{ P-value } \\
\hline & $(-)^{\star}$ in \% & $(+)^{\star}$ in \% & \\
\hline Total patients & 640770 & 25478 & - \\
\hline Mean age (SD), in years & $37.9(8.6)$ & $35.7(8.7)$ & $<0.001$ \\
\hline \multicolumn{4}{|l|}{ Gender } \\
\hline Male & 53.8 & 70.9 & \multirow{2}{*}{$<0.001$} \\
\hline Female & 46.2 & 29.1 & \\
\hline \multicolumn{4}{|l|}{ Race } \\
\hline White & 61.0 & 50.5 & \multirow{4}{*}{$<0.001$} \\
\hline Black & 17.7 & 32.6 & \\
\hline Hispanic & 15.3 & 12.1 & \\
\hline Other & 6.0 & 4.8 & \\
\hline
\end{tabular}

\section{TABLE 2: Acute pancreatitis inpatients by cannabis abuse}

*(-) refers to non-cannabis abuse and (+) refers to cannabis abuse groups.

SD: standard deviation

\section{Discussion}

Men in our study sample had 1.5 times higher odds for AP-related hospitalization than females. This may be due to increased comorbidities seen in men like diabetes, hypertension, and coronary artery disease; also, they are more likely to smoke cigarettes and abuse drugs [11]. Whites have a higher association of being hospitalized due to AP, whereas blacks, Hispanics, and other races/ethnicities didn't have any significant relationship. It may be because whites are more likely to consume alcohol, which is a potential risk factor for worsening symptoms of AP [12].

Among the risk factors, gallstones and alcohol abuse increased the likelihood of AP-related hospitalization. Gallstone exacerbates AP by increasing duct pressure, which leads to unregulated activation of digestive enzymes [13]. Ethanol and its metabolite produce oxidative stress or the generation of fatty acid ethyl esters, leading to inflammation, which may be the reason for causing acute pancreatitis [12]. Simultaneous usage of alcohol and cannabis is found to be nearly twice as common as concurrent use, suggesting that people who reported consuming both did so at the same time [6]. In our study, cystic fibrosis increased the risk by 2.5 times, as it alters the composition of pancreatic secretions, which ultimately leads to acinus plugging and dilation, causing epithelial injury and destruction accompanied by inflammation [14]. Also, hypertriglyceridemia and hypercalcemia had a higher impact on increasing the likelihood of AP-related hospitalization. Although the precise cause is not understood, the hypothesis may be that chylomicron and free fatty acids, which increase plasma viscosity, may trigger ischemia and organ inflammation [15]. It is hypothesized that hypercalcemia causes pancreatic damage by conversion of trypsinogen to trypsin [16].

In our study, it was found that cannabis abusers had twice the higher risk of association with increased hospitalization. After controlling for other risk factors, we didn't find an increase in hospitalization due to acute pancreatitis. A similar result was seen in the study where the patient with cannabis exposure had significantly lower inpatient mortality than the non-cannabis group [17]. Cannabinoids function by stimulating two receptors, cannabinoid receptor type 1 (cb1) and type 2 (cb2), within the human body's endocannabinoid system. Tetrahydrocannabinol binds to activate cannabinoid receptors, predominantly cb1 receptors in the pancreas [18-19]. Activation of cb1 receptors initiates a proinflammatory cascade leading to hypotension, tachycardia, altered consciousness, and septic shock [20-21]. Cerulein administration, a natural endogenous ligand for the cb1 receptor, leads to decreased blood flow and affecting deoxyribonucleic acid (DNA) synthesis leading to pancreatic edema and inflammatory infiltration [22]. Cannabinoids reduce blood flow by reducing blood vessel proangiogenic factors and vascular endothelial growth factors. Activation of cb1 or cb2 receptors can also inhibit adenylyl cyclase activity and decrease cyclic adenosine monophosphate levels, which ultimately affects gene transcription and cause apoptosis [18]. Basile et al. study showed a 1.2 times higher risk of post-endoscopic retrograde cholangiopancreatography pancreatitis in cannabis abusers due to tissue injury caused by cannabis [23]. A study by Matsuda et al. found that the downregulation of the endocannabinoid system by am251 (cannabinoid cb1 receptor inhibitor) with severe 
AP showed an improved survival rate in rats [24]. On the contrary, Michalski et al. study showed hu210, a synthetic agonist of cannabinoid receptors $\mathrm{cb} 1$ and $\mathrm{cb} 2$, resolved the abdominal pain induced by AP and decreased the inflammation of the pancreas, shows therapeutic benefits of the pancreas [25]. Also, a study by Barkin et al. showed cannabis could be the potential risk factor for causing AP and recurrent AP in a young population under 35 years of age [26].

The limitation of this study includes the fact that it is a cross-sectional study and, hence, cannot establish or deny any causal relationship between AP and cannabis abuse. The NIS is administrative data, lacks patientlevel clinical information, and sampling was based on diagnostic codes, which may have resulted in underreporting of comorbidities and is subject to prevalence-incidence bias. So, there exists an information bias due to a lack of details on the amount, dose, frequency, and last episodic use of cannabis for the study in patients, which can affect the association/relationship with AP hospitalization. Yet, the NIS offers the largest inpatient data pool in the US. Hence, the data is incomparable to the population-based perception of disease associations with systematic and temporal factors. The sample size is very large and is diverse and represents the inpatient population across the US.

\section{Conclusions}

Cannabis abuse does not independently increase the odds of AP-related hospitalization. Cannabis-induced AP can be treated if a persistent cannabis abuse pattern is discontinued at an earlier stage. Therefore, awareness campaigns and early interventional supportive therapies among cannabis abusers might help diagnose and treat comorbid cannabis and other substance-use disorders and improve the quality of life. Many studies concerning the pathophysiology of cannabis abuse and AP are required in both animal and human models to identify the impact and also to address and treat persistent cannabis abuse patterns on gastrointestinal health.

\section{Additional Information \\ Disclosures}

Human subjects: Consent was obtained or waived by all participants in this study. Animal subjects: All authors have confirmed that this study did not involve animal subjects or tissue. Conflicts of interest: In compliance with the ICMJE uniform disclosure form, all authors declare the following: Payment/services info: All authors have declared that no financial support was received from any organization for the submitted work. Financial relationships: All authors have declared that they have no financial relationships at present or within the previous three years with any organizations that might have an interest in the submitted work. Other relationships: All authors have declared that there are no other relationships or activities that could appear to have influenced the submitted work.

\section{References}

1. Peery AF, Crockett SD, Barritt AS, et al.: Burden of gastrointestinal, liver, and pancreatic diseases in the United States. Gastroenterology. 2015, 149:1731-1741.e3. 10.1053/j.gastro.2015.08.045

2. Yadav D, Lowenfels AB: Trends in the epidemiology of the first attack of acute pancreatitis. A systematic review. Pancreas. 2006, 33:323-30. 10.1097/01.mpa.0000236733.31617.52

3. Xiao AY, Tan ML, Wu LM, et al.: Global incidence and mortality of pancreatic diseases: a systematic review, meta-analysis, and meta-regression of population-based cohort studies. Lancet Gastroenterol Hepatol. 2016, 1:45-5. 10.1016/S2468-1253(16)30004-8

4. Yu B, Chen X, Chen X, Yan H: Marijuana legalization and historical trends in marijuana use among US residents aged 12-25: results from the 1979-2016 National Survey on drug use and health. BMC Public Health. 2020, 20:156. 10.1186/s12889-020-8253-4

5. Cohen K, Weizman A, Weinstein A: Positive and negative effects of cannabis and cannabinoids on health . Clin Pharmacol Ther. 2019, 105:1139-47. 10.1002/cpt.1381

6. Subbaraman MS, Kerr WC: Simultaneous versus concurrent use of alcohol and cannabis in the National Alcohol Survey. Alcohol Clin Exp Res. 2015, 39:872-9. 10.1111/acer.12698

7. Goyal H, Guerreso K, Smith B, Harper K, Patel S, Patel A, Parikh P: Severity and outcomes of acute alcoholic pancreatitis in cannabis users. Transl Gastroenterol Hepatol. 2017, 2:60. 10.21037/tgh.2017.06.03

8. Pagliari D, Saviano A, Brizi MG, et al.: Cannabis-induced acute pancreatitis: a case report with comprehensive literature review. Eur Rev Med Pharmacol Sci. 2019, 23:8625-9. 10.26355/eurrev 20191019179

9. Overview of the national (nationwide) inpatient sample . (2021). Accessed: May 1, 2021: https://www.hcupus.ahrq.gov/nisoverview.jsp.

10. NIS description of data elements. (2021). Accessed: May 1, 2021: https://www.hcupus.ahrq.gov/db/nation/nis/nisdde.jsp.

11. Sharma P, Aguilar R, Nader MA, et al.: Weekend effect in acute pancreatitis-related hospital admissions in the United States. An analysis of the nationwide inpatient sample. Pancreas. 2018, 47:418-24. 10.1097/MPA.0000000000001008

12. Bhandari R, Khaliq K, Ravat V, Kaur P, Patel RS: Chronic alcoholic liver disease and mortality risk in spontaneous bacterial peritonitis: analysis of 6,530 hospitalizations. Cureus. 2020, 12:e8189. 10.7759 /cureus.8189

13. Wang GJ, Gao CF, Wei D, Wang C, Ding SQ: Acute pancreatitis: etiology and common pathogenesis . World J Gastroenterol. 2009, 15:1427-30. 10.3748/wjg.15.1427 
14. Gibson-Corley KN, Meyerholz DK, Engelhardt JF: Pancreatic pathophysiology in cystic fibrosis. J Pathol. 2016, 238:311-20. 10.1002/path.4634

15. de Pretis N, Amodio A, Frulloni L: Hypertriglyceridemic pancreatitis: epidemiology, pathophysiology and clinical management. United European Gastroenterol J. 2018, 6:649-55. 10.1177/2050640618755002

16. Kumar A, Kumar PG, Pujahari AK, Sampath S: Hypercalcemia related pancreatitis. Med J Armed Forces India. 2010, 66:385-6. 10.1016/S0377-1237(10)80025-5

17. Simons-Linares CR, Barkin JA, Jang S, et al.: The impact of cannabis consumption on mortality, morbidity, and cost in acute pancreatitis patients in the United States. A 10-year analysis of the national inpatient sample. Pancreas. 2019, 48:850-5. 10.1097/MPA.0000000000001343

18. Sharafi G, He H, Nikfarjam M: Potential use of cannabinoids for the treatment of pancreatic cancer . J Pancreat Cancer. 2019, 5:1-7. 10.1089/pancan.2018.0019

19. Bermúdez-Silva FJ, Suárez J, Baixeras E, et al.: Presence of functional cannabinoid receptors in human endocrine pancreas. Diabetologia. 2008, 51:476-87. 10.1007/s00125-007-0890-y

20. Varga K, Wagner JA, Bridgen DT, Kunos G: Platelet- and macrophage-derived endogenous cannabinoids are involved in endotoxin-induced hypotension. FASEB J. 1998, 12:1035-44. 10.1096/fasebj.12.11.1035

21. Devane WA, Hanus L, Breuer A, et al.: Isolation and structure of a brain constituent that binds to the cannabinoid receptor. Science. 1992, 258:1946-9. 10.1126/science.1470919

22. Dembiński A, Warzecha Z, Ceranowicz P, et al.: Cannabinoids in acute gastric damage and pancreatitis . J Physiol Pharmacol. 2006, 57 Suppl 5:137-54.

23. Njei B, Sharma P, McCarty TR, et al.: Cannabis use is associated with increased risk of post-endoscopic retrograde cholangiopancreatography pancreatitis. Analysis of the US Nationwide Inpatient Sample Database, 2004-2014. Pancreas. 2018, 47:1142-9. 10.1097/MPA.0000000000001143

24. Matsuda K, Mikami Y, Takeda K, et al.: The cannabinoid 1 receptor antagonist, AM251, prolongs the survival of rats with severe acute pancreatitis. Tohoku J Exp Med. 2005, 207:99-107. 10.1620/tjem.207.99

25. Michalski CW, Laukert T, Sauliunaite D, et al.: Cannabinoids ameliorate pain and reduce disease pathology in cerulein-induced acute pancreatitis. Gastroenterology. 2007, 132:1968-78. 10.1053/j.gastro.2007.02.035

26. Barkin JA, Nemeth Z, Saluja AK, Barkin JS: Cannabis-induced acute pancreatitis: a systematic review . Pancreas. 2017, 46:1035-8. 10.1097/MPA.0000000000000873 